CUQM-94

HEPHY-PUB 760/02

UWThPh-2002-19

hep-th/0210149

October 2002

\title{
DISCRETE SPECTRA OF SEMIRELATIVISTIC HAMILTONIANS
}

\author{
Richard L. HALL \\ Department of Mathematics and Statistics, Concordia University, \\ 1455 de Maisonneuve Boulevard West, Montréal, Québec, Canada H3G 1M8 \\ Wolfgang LUCHA* \\ Institut für Hochenergiephysik, \\ Österreichische Akademie der Wissenschaften, \\ Nikolsdorfergasse 18, A-1050 Wien, Austria \\ Franz F. SCHÖBERL ${ }^{\dagger}$ \\ Institut für Theoretische Physik, Universität Wien, \\ Boltzmanngasse 5, A-1090 Wien, Austria
}

\begin{abstract}
We review various attempts to localize the discrete spectra of semirelativistic Hamiltonians of the form $H=\beta \sqrt{m^{2}+\mathbf{p}^{2}}+V(r)$ (defined, without loss of generality but for definiteness, in three spatial dimensions) as entering, for instance, in the spinless Salpeter equation; every Hamiltonian in this class of operators consists of the relativistic kinetic energy $\beta \sqrt{m^{2}+\mathbf{p}^{2}}$, where $\beta>0$ allows for the possibility of more than one particles of mass $m$, and a spherically symmetric attractive potential $V(r), r \equiv|\mathbf{x}|$. In general, accurate eigenvalues of a nonlocal Hamiltonian operator can only be found by the use of a numerical approximation procedure. Our main emphasis, however, is on the derivation of rigorous semi-analytical expressions for both upper and lower bounds to the energy levels of such operators. We compare the bounds obtained within different approaches and present relationships existing between the bounds.
\end{abstract}

PACS numbers: 03.65.Ge, 03.65.Pm, 11.10.St

$\ddagger$ E-mail address: rhall@mathstat.concordia.ca

* E-mail address: wolfgang.lucha@oeaw.ac.at

† E-mail address: franz.schoeberl@univie.ac.at 


\section{Introduction: The "spinless-Salpeter" Hamiltonian}

We investigate the discrete spectrum - that is, the set of eigenvalues $E_{n \ell}$ below the onset of the essential spectrum, corresponding to bound states characterized by the radial quantum number $n=1,2,3, \ldots$ and the angular-momentum quantum number $\ell=0,1,2, \ldots$ of the semirelativistic "spinless-Salpeter" Hamiltonian for particles of mass $m$ and momentum $\mathbf{p}$,

$$
H=\beta \sqrt{m^{2}+\mathbf{p}^{2}}+V(r), \quad r \equiv|\mathbf{x}|, \quad \beta>0,
$$

where $V(r)$ is an attractive central potential in three spatial dimensions. As is evident from the presence of the relativistic kinetic-energy operator $\beta \sqrt{m^{2}+\mathbf{p}^{2}}$ (with $\beta>0$ allowing, e.g., for more than one particle), this Hamiltonian may be regarded as the generalization of the nonrelativistic Schrödinger Hamiltonian towards relativistic kinematics. The eigenvalue equation of the operator (1), called the "spinless Salpeter equation," arises as a well-defined approximation to the Bethe-Salpeter formalism for the description of bound states within a relativistic quantum field theory. Our main goal is to discuss bounds on the energy levels $E$.

\section{Envelope theory for energy bounds}

The envelope theory, developed in Refs. [1,2], constructs bounds on the discrete eigenvalues $E_{n \ell}$ of a given operator $H$ by comparing the spectrum of $H$ with the spectrum of a suitably formulated "tangential problem," for which some spectral information is known (or may be obtained with considerable more ease than for the original problem). More specifically, when applied to the (semirelativistic) Hamiltonian $H$ defined in Eq. (1) [3, 4], the envelope theory compares $H$ with a corresponding "tangential Hamiltonian"

$$
\widetilde{H}=\beta \sqrt{m^{2}+\mathbf{p}^{2}}+v h(r), \quad v>0,
$$

involving some "basis potential" $h(r)$. Under the assumption that the interaction potential under consideration, $V(r)$, is a smooth transformation $V(r)=g(h(r))$ of the basis potential $h(r)$, where the function $g(h)$ has definite convexity, this comparison yields rigorous bounds on the discrete eigenvalues $E_{n \ell}$ of $H$. More precisely, if the function $g(h)$ is convex, that is, $g^{\prime \prime}(h)>0$, we obtain lower energy bounds; if the function $g(h)$ is concave, that is, $g^{\prime \prime}(h)<0$, we obtain upper energy bounds. If the eigenvalues $\widetilde{E}$ of $\widetilde{H}$ are not known (analytically), the envelope theory may take advantage of the knowledge of suitable bounds on the eigenvalues, namely, of lower bounds on $\widetilde{E}_{n \ell}$ for convex $g(h)$ or of upper bounds on $\widetilde{E}_{n \ell}$ for concave $g(h)$.

Suppressing the quantum numbers $n, \ell$ identifying the bound state under consideration, we denote, for a given value of the coupling $v$ entering into the "tangential Hamiltonian" $\widetilde{H}$, the eigenvalues of $\widetilde{H}$ or the appropriate - in the above sense - bounds to the latter by $e(v)$. The strict bounds on the discrete eigenvalues $E$ of $H$ predicted within the framework of the envelope technique may then be summarized in form of the "principal envelope formula" [4]

$$
E \approx \min _{v>0}\left[e(v)-v e^{\prime}(v)+g\left(e^{\prime}(v)\right)\right],
$$

where the sign of approximate equality is used to recall that, for a definite convexity of $g(h)$, the envelope theory yields lower bounds for convex $g(h)$ and upper bounds for concave $g(h)$.

For the tangential problem posed by the (semirelativistic) "tangential Hamiltonian" $\widetilde{H}$, spectral information is, at present, available if the basis potential $h(r)$ is either the Coulomb potential, that is, $h(r)=-1 / r$, or the harmonic-oscillator potential, that is, $h(r)=r^{2}$. The energy bounds resulting in these two cases are discussed, in turn, in Sec. 4 and Sec. 5. The case of the interaction potential $V(r)$ in Eq. (1) being the harmonic-oscillator potential may be traced back to a nonrelativistic Schrödinger problem; this is studied for its own in Sec. 3. 


\section{Harmonic-oscillator potential}

The "relativistic harmonic-oscillator problem," defined by the special case of $V(r)$ being the harmonic-oscillator potential $V(r)=v r^{2}$, may be analyzed by observing $[5,6,7,8,9]$ that in momentum-space representation the semirelativistic Hamiltonian $H=\beta \sqrt{m^{2}+\mathbf{p}^{2}}+v r^{2}$ of Eq. (1) simplifies to the (nonrelativistic) Schrödinger operator $\mathcal{H}$ given by

$$
\mathcal{H}=-v \Delta+\mathcal{V}(r), \quad \mathcal{V}(r)=\beta \sqrt{m^{2}+r^{2}} .
$$

Thus the eigenvalue equation for $H$ reduces to an easier-to-treat nonrelativistic Schrödinger problem with an effective interaction potential $\mathcal{V}(r)$ which is reminiscent of the square root.

\subsection{Upper bounds on harmonic-oscillator energy levels}

The potential $\mathcal{V}$ is a concave transform of a harmonic-oscillator potential. Thus we may find upper bounds on the eigenvalues $E_{n \ell}(v)$ of $\mathcal{H}$ (cf. Eq. (2.2) of Ref. [3] or Eq. (12) of Ref. [4]):

$$
E_{n \ell}(v) \leq \min _{r>0}\left[\beta \sqrt{m^{2}+\frac{P_{n \ell}^{2}(2)}{r^{2}}}+v r^{2}\right], \quad P_{n \ell}(2)=2 n+\ell-\frac{1}{2} .
$$

The (dimensionless) numbers $P_{n \ell}(2)$ introduced here are related to the eigenvalues $\mathcal{E}_{n \ell}$ of the Hamiltonian $\widetilde{H}=\mathbf{p}^{2}+v r^{2}$ by $\mathcal{E}_{n \ell}=2 \sqrt{v} P_{n \ell}(2)$.

Upper bounds on the eigenvalues of self-adjoint operators bounded from below may also be found by combining the minimum-maximum principle $[10,11]$ with appropriate operator inequalities. (For details, see, e.g., Sec. 3.4 of Ref. [5], Sec. 3.5 of Ref. [6], Sec. 2.4 of Ref. [7], or Appendix A of Ref. [12].) Exploiting the obvious positivity of $\left(\sqrt{m^{2}+\mathbf{p}^{2}}-\mu\right)^{2}$, where $\mu$ is an arbitrary real parameter (with the dimension of mass), we obtain a set of inequalities for the square-root operator $\sqrt{m^{2}+\mathbf{p}^{2}}$ entering into the kinetic energy of the Hamiltonian (1); cf. also Ref. [13]:

$$
\sqrt{m^{2}+\mathbf{p}^{2}} \leq \frac{\mathbf{p}^{2}+m^{2}+\mu^{2}}{2 \mu} \quad \forall \mu>0
$$

(This operator inequality may also be found by constructing the tangent line $a\left(\mathbf{p}_{1}^{2}\right) \mathbf{p}^{2}+b\left(\mathbf{p}_{1}^{2}\right)$ to the square root $\sqrt{m^{2}+\mathbf{p}^{2}}$ at the point of contact $\mathbf{p}_{1}^{2}=\mu^{2}-m^{2}$; see Sec. 4 of Ref. [4].) Amending this result by the interaction potential $V(r)$, one finds that the spinless-Salpeter Hamiltonian (1) satisfies

$$
H \leq H_{\mathrm{NR}}(\mu) \equiv \beta \frac{\mathbf{p}^{2}+m^{2}+\mu^{2}}{2 \mu}+V(r) \quad \forall \mu>0 .
$$

The min-max principle then tells us that the discrete eigenvalues $E_{n \ell}$ of the semirelativistic Hamiltonian $H$ of Eq. (1) are bounded from above by the corresponding discrete eigenvalues $E_{\mathrm{NR}, n \ell}(\mu)$ of the nonrelativistic Hamiltonian $H_{\mathrm{NR}}(\mu)$ and consequently also by the minimum $\bar{E}_{n \ell}$ with respect to $\mu$ of all these upper bounds [5]:

$$
E_{n \ell} \leq \bar{E}_{n \ell} \equiv \min _{\mu>0} E_{\mathrm{NR}, n \ell}(\mu) .
$$

Applying these general findings to the case of the harmonic-oscillator potential, one ends up with the following upper bounds for all energy levels of the relativistic harmonic-oscillator problem (see Appendix A of Ref. [9]; for the ease of comparison, we express here the bounds of Ref. [9] in terms of $P_{n \ell}(2)$ rather than in terms of $\left.E_{\mathrm{NR}, n \ell}(\mu)\right)$ :

$$
E_{n \ell}(v) \leq \bar{E}_{n \ell} \equiv \min _{\mu>0}\left[\beta \frac{m^{2}+\mu^{2}}{2 \mu}+\sqrt{\frac{2 \beta v}{\mu}} P_{n \ell}(2)\right], \quad P_{n \ell}(2)=2 n+\ell-\frac{1}{2} .
$$


Despite their seemingly different form, the upper bounds in Eqs. (4) and (5) are, in fact, identical, in the sense that the two functions on the right-hand side of these inequalities have their minimum at the same critical point and take the same miminal value at this point.

In order to demonstrate this equivalence, let us start from the "min-max" expression (5) for these upper bounds. For simplicity of notation, we suppress for the moment the quantum numbers $n, \ell$ which identify the bound states under consideration. The upper bound $\bar{E}$ then reads

$$
\bar{E}=\min _{\mu>0}\left[\beta \frac{m^{2}+\mu^{2}}{2 \mu}+\sqrt{\frac{\beta v}{2 \mu}} 2 P\right] .
$$

We introduce the abbreviation $q=p^{2}$, define the function $f(q)=\sqrt{m^{2}+q}$, which satisfies

$$
f^{\prime}(q)=\frac{1}{2 f(q)}, \quad f^{2}(q)=m^{2}+q
$$

and identify the (positive but otherwise arbitrary) parameter $\mu$ according to $\mu=f(q)$. Next, we introduce a function $F$ by the definition $F(v)=2 P \sqrt{v}$. A couple of algebraic steps then recasts the upper bound $\bar{E}$ into the form

$$
\bar{E}=\min _{q>0}\left[F\left(\beta v f^{\prime}\right)+\beta\left(f-q f^{\prime}\right)\right] .
$$

The critical points of this latter expression are obtained by differentiation with respect to $q$. Since $f^{\prime \prime}(q) \neq 0$, this yields

$$
\hat{q}=v F^{\prime}\left(\beta v f^{\prime}(\hat{q})\right)
$$

and

$$
\bar{E}=F\left(\beta v f^{\prime}(\hat{q})\right)-\beta v f^{\prime}(\hat{q}) F^{\prime}\left(\beta v f^{\prime}(\hat{q})\right)+\beta f(\hat{q}) .
$$

Let us define a new problem, related to the above one by a Legendre transformation [14], which has the same minimum and the same critical point, but the function to be minimized is different. Let $u(q)=\beta v f^{\prime}(q)>0$ and consider the energy expression

$$
\hat{E}=\min _{u>0}\left[F(u)-u F^{\prime}(u)+\beta f\left(v F^{\prime}(u)\right)\right] .
$$

Since $F^{\prime \prime}(u) \neq 0$, we find that the critical point is given by $\hat{u}=\beta v f^{\prime}\left(v F^{\prime}(\hat{u})\right)$. But, given the definition of $u$, this equation is equivalent to the earlier critical equation $\hat{q}=v F^{\prime}\left(\beta v f^{\prime}(\hat{q})\right)$. Meanwhile, the critical value $\hat{E}$ is also the same as the critical value $\bar{E}$ because we have

$$
\hat{E}=F\left(\beta v f^{\prime}(\hat{q})\right)-\beta v f^{\prime}(\hat{q}) F^{\prime}\left(\beta v f^{\prime}(\hat{q})\right)+\beta f(\hat{q})=\bar{E} .
$$

Having shown that $\bar{E}$ yields the same results as $\hat{E}$, we now transform $\hat{E}$ into the form (4). First of all, we note that $F(u)-u F^{\prime}(u)=P \sqrt{u}$. Next, we define a new dummy minimization variable $r>0$ by

$$
\sqrt{u}=\frac{v r^{2}}{P}
$$

From this, it follows that

$$
F^{\prime}(u)=\frac{P}{\sqrt{u}}=\frac{P^{2}}{v r^{2}} .
$$

Finally, remembering the definition $f(q)=\sqrt{m^{2}+q}$ of $f(q)$, we may transform $\hat{E}$ into the expression on the right-hand side of the inequality (4); this establishes the equivalence of the upper bounds (4) and (5). 


\subsection{Lower bounds on harmonic-oscillator energy levels}

The potential $\mathcal{V}$ in Eq. (3) is a convex transform of a linear potential. Therefore we may find lower bounds on the eigenvalues $E_{n \ell}(v)$ of $\mathcal{H}$ (cf. Eq. (2.2) of Ref. [3] or Eq. (12) of Ref. [4]):

$$
E_{n \ell}(v) \geq \min _{r>0}\left[\beta \sqrt{m^{2}+\frac{P_{n \ell}^{2}(1)}{r^{2}}}+v r^{2}\right],
$$

where the (dimensionless) numbers $P_{n \ell}(1)$ introduced here are related to the eigenvalues $\mathcal{E}_{n \ell}$ of the Hamiltonian $\widetilde{H}=\mathbf{p}^{2}+v r$ by

$$
\mathcal{E}_{n \ell}=3\left[\frac{v P_{n \ell}(1)}{2}\right]^{2 / 3} ;
$$

for the lowest states $(n=1,2, \ldots, 5, \ell=0,1, \ldots, 5)$ these numbers may be found in Table 1 .

Table 1: Numerical values of the energy-related numbers $P_{n \ell}(1)$ for the lowest energy levels.

\begin{tabular}{ccc}
\hline \hline$n$ & $\ell$ & $P_{n \ell}(1)$ \\
\hline 1 & 0 & 1.37608 \\
2 & 0 & 3.18131 \\
3 & 0 & 4.99255 \\
4 & 0 & 6.80514 \\
5 & 0 & 8.61823 \\
1 & 1 & 2.37192 \\
2 & 1 & 4.15501 \\
3 & 1 & 5.95300 \\
4 & 1 & 7.75701 \\
5 & 1 & 9.56408 \\
\hline \hline
\end{tabular}

\begin{tabular}{rrr}
\hline \hline$n$ & $\ell$ & \multicolumn{1}{c}{$P_{n \ell}(1)$} \\
\hline 1 & 2 & 3.37018 \\
2 & 2 & 5.14135 \\
3 & 2 & 6.92911 \\
4 & 2 & 8.72515 \\
5 & 2 & 10.52596 \\
1 & 3 & 4.36923 \\
2 & 3 & 6.13298 \\
3 & 3 & 7.91304 \\
4 & 3 & 9.70236 \\
5 & 3 & 11.49748 \\
\hline \hline
\end{tabular}

\begin{tabular}{rrr}
\hline \hline$n$ & $\ell$ & \multicolumn{1}{c}{$P_{n \ell}(1)$} \\
\hline 1 & 4 & 5.36863 \\
2 & 4 & 7.12732 \\
3 & 4 & 8.90148 \\
4 & 4 & 10.68521 \\
5 & 4 & 12.47532 \\
1 & 5 & 6.36822 \\
2 & 5 & 8.12324 \\
3 & 5 & 9.89276 \\
4 & 5 & 11.67183 \\
5 & 5 & 13.45756 \\
\hline \hline
\end{tabular}

Lower bounds on the eigenvalues of (self-adjoint) Hamiltonians involving the relativistic kinetic energy $\sqrt{m^{2}+\mathrm{p}^{2}}$ may also be found by taking into account the obvious positivity of $\left[\mathbf{p}^{2} \xi^{2}-m^{2}\left(1-\xi^{2}\right)\right]^{2}$ for an arbitrary real parameter $\xi$, which may be converted into the set of operator inequalities

$$
\sqrt{m^{2}+\mathbf{p}^{2}} \geq|\mathbf{p}| \sqrt{1-\xi^{2}}+m \xi, \quad 0 \leq \xi \leq 1 .
$$

Applying these general findings to the semirelativistic Hamiltonian $H$ of Eq. (1), with $V(r)$ being the harmonic-oscillator potential, we obtain lower bounds on all discrete energy levels $E_{n \ell}$ of the relativistic harmonic-oscillator problem (for details, see Appendix A of Ref. [9]):

$$
E_{n \ell}(v) \geq \underline{E}_{n \ell} \equiv \max _{0 \leq \xi \leq 1}\left[\beta \xi m+\beta^{2 / 3}\left(1-\xi^{2}\right)^{1 / 3} E_{n \ell}(v ; m=0)\right],
$$

where $E_{n \ell}(v ; m=0)$ denote the corresponding discrete eigenvalues of the operator $|\mathbf{p}|+v r^{2}$, i.e., of the Hamiltonian (1) for $\beta=1$ and a vanishing particle mass $m$. For states with $\ell=0$, for instance, these "zero-mass" energy levels are given, in terms of the zeros $z_{n}$ of the Airy function $\operatorname{Ai}(z)[15]\left(-z_{n}=2.33810,4.08794,5.52055, \ldots\right)$, by $E_{n 0}(v ; m=0)=-v^{1 / 3} z_{n}[9]$. 
As in Sec. 3.1 we observe that the lower bounds in Eqs. (6) and (8) appear, at first sight, to be rather different. A closer inspection, however, reveals that these bounds are identical.

In order to present an analytical proof of the equivalence of the lower bounds (6) and (8) on the energy eigenvalues of the relativistic harmonic-oscillator problem, we recall that the envelope lower bounds in (6) are based (in the momentum-space representation of $H$ ) on the observation that, since its second derivative with respect to $|\mathbf{p}|$ is positive, the square-root operator $\sqrt{m^{2}+\mathbf{p}^{2}}$ of the relativistic kinetic energy is bounded from below by tangent lines $a(t)+b(t)|\mathbf{p}|$; this may be expressed by the geometrical inequality $\sqrt{m^{2}+\mathbf{p}^{2}} \geq a(t)+b(t)|\mathbf{p}|$, where, by elementary calculus,

$$
a(t)=\frac{m^{2}}{\sqrt{m^{2}+t^{2}}}, \quad b(t)=\frac{t}{\sqrt{m^{2}+t^{2}}},
$$

and $|\mathbf{p}|=t$ is the point of contact between the square root of the relativistic kinetic energy and its straight-line lower approximation. The equivalence of the above two expressions for the harmonic-oscillator lower bound is then shown by a simple change of variable from $t$ to $\xi$ given explicitly by

$$
\xi=\frac{a(t)}{m},
$$

which, after a little algebra, leads to $b(t)=\sqrt{1-\xi^{2}}$. For these coefficient functions $a(t)$ and $b(t)$ the above geometrical inequality becomes exactly the lower bound (7) on the relativistic kinetic energy. Consequently, with the basic inequalities being identical, the resulting lower energy bounds have to be identical too.

\section{Convex transform of Coulomb potential}

For the "relativistic Coulomb problem" posed by the spinless-Salpeter Hamiltonian (1) with $V(r)$ being the Coulomb potential $V(r)=-v / r$, for coupling constants $v$ smaller than $\beta v_{\mathrm{c}}$, that is, $v<\beta v_{\mathrm{c}}$, where the critical value $v_{\mathrm{c}}$ of the Coulomb coupling constant $v$ is given by

$$
v_{\mathrm{c}}=\frac{2}{\pi},
$$

a lower bound to the ground-state energy eigenvalue $E_{0}$ of $H$ (the bottom of the spectrum of $H$ ) has been derived by Herbst [16]:

$$
E_{0} \geq \beta m \sqrt{1-\left(\frac{\sigma v}{\beta}\right)^{2}}, \quad \sigma \equiv \frac{\pi}{2} .
$$

For some part of the allowed range of the coupling $v$, this lower bound has been improved by Martin and Roy [17]:

$$
E_{0} \geq \beta m \sqrt{\frac{1}{2}\left[1+\sqrt{1-\left(\frac{2 v}{\beta}\right)^{2}}\right]}, \quad v<\frac{\beta}{2} .
$$

In Ref. [4], we recast this Martin-Roy bound into a class of lower bounds on the spectrum of $H$ all of which are of the form of Herbst's bound (9) [but, of course, slightly weaker than the Martin-Roy bound (10)]; the new lower bounds are parametrized by a real parameter $\sigma \geq 1$ :

$$
E_{0} \geq \beta m \sqrt{1-\left(\frac{\sigma v}{\beta}\right)^{2}}, \quad v \leq \beta \frac{\sqrt{\sigma^{2}-1}}{\sigma^{2}}<\frac{\beta}{2} .
$$


The constraint on the Coulomb coupling $v$ in Eq. (11) automatically guarantees $v \leq \beta / \sigma$; this latter constraint is a necessary and sufficient condition for the reality of the lower energy bounds in Eq. (11).

From the lower bounds (9) or (11) on the spectrum of the relativistic Coulomb problem, for the class of potentials $V(r)$ which are convex transforms $V(r)=g(h(r)), g^{\prime \prime}>0$, of the Coulomb potential $h(r)=-1 / r$ an ("absolute") envelope lower bound on the ground-state eigenvalue $E_{0}$ (and, hence, on the entire spectrum) of the spinless-Salpeter Hamiltonian (1) may be found (cf. Eq. (16) of Ref. [4]):

$$
E_{0} \geq \min _{r>0}\left[\beta \sqrt{m^{2}+\frac{P^{2}}{r^{2}}}+V(r)\right], \quad v<\beta v_{P} .
$$

Here we introduced a parameter $P$ by defining $P=1 / \sigma$. Accordingly, the boundary value $v_{P}$ of the Coulomb coupling constant $v$ is given, when arising from demanding the Hamiltonian (1) to be bounded from below, by the critical coupling $v_{\mathrm{c}}$, i.e., $v_{P}=v_{\mathrm{c}}$, or, when arising from the region of validity of our new family of Coulomb lower bounds (11), by

$$
v_{P}=P \sqrt{1-P^{2}}<\frac{1}{2} .
$$

It is easy to convince oneself that the Coulomb lower energy bound on the right-hand side of the inequality (12) is a monotone increasing function $\underline{E}(P)$ of the parameter $P$. To this end, we rewrite the inequality (12) in the form

$$
E_{0} \geq \underline{E}(P)=\min _{r>0} F(r, P)
$$

with

$$
F(r, P) \equiv \beta \sqrt{m^{2}+\frac{P^{2}}{r^{2}}}+V(r) .
$$

Remembering that $V(r)$ is the transform $V(r)=g(h(r))$ of $h(r)=-1 / r$, and assuming that $g^{\prime}(h)>0$, the critical point $\hat{r}(P)$ (corresponding to the minimum of $F(r, P)$ for a given $P$ ) may be found, from

$$
\left.\frac{\partial F(r, P)}{\partial r}\right|_{r=\hat{r}(P)}=0
$$

by solving

$$
\frac{\beta P^{2}}{\sqrt{m^{2} \hat{r}^{2}+P^{2}}}=g^{\prime}(h(\hat{r})) .
$$

The substitution of the solution $\hat{r}(P)$ of the latter equation in $F(r, P)$ gives the lower bound $\underline{E}(P)=F(\hat{r}(P), P)$; here we are interested in the total derivative of $\underline{E}(P)$ with respect to $P$. Formally, this derivative would be given by

$$
\frac{\mathrm{d} \underline{E}(P)}{\mathrm{d} P}=\frac{\partial F(\hat{r}(P), P)}{\partial P}+\left.\frac{\partial F(r, P)}{\partial r}\right|_{r=\hat{r}(P)} \frac{\mathrm{d} \hat{r}(P)}{\mathrm{d} P}
$$

At the critical point, however, the first factor of the second term vanishes and one is left with

$$
\frac{\mathrm{d} \underline{E}(P)}{\mathrm{d} P}=\frac{\partial F(\hat{r}(P), P)}{\partial P}=\frac{\beta P}{\hat{r} \sqrt{m^{2} \hat{r}^{2}+P^{2}}}>0 .
$$

From this we conclude that the lower bound $\underline{E}(P)$ is a monotone increasing function of $P$. 
The existence of the upper bound $v<\beta v_{P}<\beta / 2$ on the Coulomb coupling constant $v$ is reflected in a corresponding constraint on the parameters of the interaction potential $V(r)$. The envelope theory constructs a "tangential potential" to $V(r)$ at a point of contact $r=t$. For a potential $V(r)$ which is a convex transform $V(r)=g(h(r))$ of the basis potential $h(r)$, this tangential potential forms a lower bound to $V(r)$; for $h(r)$ being the Coulomb potential, $h(r)=-1 / r$, this lower bound is a Coulomb potential with the effective coupling constant $v(t)=t^{2} V^{\prime}(t)$, shifted by the constant $V(t)+t V^{\prime}(t)$ :

$$
V(r) \geq \widetilde{V}(r) \equiv-\frac{t^{2} V^{\prime}(t)}{r}+V(t)+t V^{\prime}(t)
$$

The relativistic Coulomb problem is posed by the "Coulombic" Hamiltonian

$$
H_{\mathrm{C}}(v)=\beta \sqrt{m^{2}+\mathbf{p}^{2}}-\frac{v}{r}, \quad v<\beta v_{\mathrm{c}}
$$

which is nothing else but the tangential Hamiltonian $\widetilde{H}$ with $h(r)=-1 / r$. In the notation of Sec. 2, the eigenvalues $E_{\mathrm{C}}(v)$ of $H_{\mathrm{C}}(v)$ are bounded from below according to $E_{\mathrm{C}}(v) \geq e(v)$, with $e(v)$ given by Herbst's bound (9), our new bound (11), or the Martin-Roy bound (10). Expressed in terms of the Coulombic Hamiltonian $H_{\mathrm{C}}(v)$, the semirelativistic Hamiltonian (1) thus satisfies the inequality $H \geq H_{\mathrm{C}}(v(t))+V(t)+t V^{\prime}(t)$; its eigenvalues $E$ are bounded from below according to

$$
E_{0} \geq E_{\mathrm{C}, 0}(v(t))+V(t)+t V^{\prime}(t) \geq e(v(t))+V(t)+t V^{\prime}(t) .
$$

The optimized lower bound on the discrete spectrum of $H$ is then found by maximizing the expression on the right-hand side of this latter inequality with respect to the point of contact $r=t$. The equation which determines the critical point $\hat{t}$ has to be solved by observing that, at the critical point $r=\hat{t}$, the effective Coulomb coupling $v(t)$ still has to satisfy $v(\hat{t})<\beta v_{P}$; this nontrivial requirement imposes the announced "Coulomb coupling constant constraint" on the coupling parameters entering in the potential $V(r)$.

It goes without saying that, when applying our "Herbst-based" lower energy bounds of Eq. (11), care has to be taken in order to assure that the chosen values of the parameters in the potential $V(r)$ under consideration respect this Coulomb coupling constant constraint.

Let us illustrate these general considerations by applying them to a Coulomb-plus-linear or (in view of its shape) "funnel" potential

$$
V(r)=-\frac{c_{1}}{r}+c_{2} r, \quad c_{1} \geq 0, \quad c_{2} \geq 0 .
$$

In this case, the effective Coulomb coupling $v(t)$ explicitly reads $v(t)=c_{1}+c_{2} t^{2}$ and, for the set of "Herbst-like" lower energy bounds $e(v)$ given by Eqs. (9) and (11), the critical point $\hat{t}$ is, after some algebra, determined by the relation

$$
v(\hat{t})=\frac{\beta P^{2}}{\sqrt{m^{2} \hat{t}^{2}+P^{2}}} .
$$

The above Coulomb coupling constant constraint becomes an upper bound on a particular linear combination of the two involved coupling parameters $c_{1}$ and $c_{2}$ (cf. Sec. 6 of Ref. [4]):

$$
c_{1}+\frac{P^{2}}{m^{2}}\left(\frac{P^{2}}{v_{P}^{2}}-1\right) c_{2}<\beta v_{P}
$$


The Coulomb lower bounds (12) on the spectrum of the semirelativistic Hamiltonian (1) may be optimized by seeking that value of the parameter $P=1 / \sigma$ which, for given values of the mass $m$ of the bound-state constituent(s) and of the coupling parameters $c_{1}$ and $c_{2}$ in the Coulomb-plus-linear potential (14), solves the Coulomb coupling constant constraint (15). Taking into account the definition (13) of the boundary value $v_{P}$ of the Coulomb coupling $v$, this optimum value of $P$ is obtained as the solution of the relation

$$
\frac{c_{2} \sin ^{4} t}{\cos ^{2} t\left(\beta \sin t \cos t-c_{1}\right)}=m^{2}, \quad P \equiv \sin t .
$$

The set (12) of envelope lower bounds distinguished from each other by the parameter $P$ may be improved somewhat by taking into account the Martin-Roy lower bound (10) on the energy spectrum of the relativistic Coulomb problem. To this end, let us denote the energy bound on the right-hand side of the inequality (10) by $e(v)$ :

$$
e(v)=\beta m \sqrt{\frac{1}{2}\left[1+\sqrt{1-\left(\frac{2 v}{\beta}\right)^{2}}\right]} .
$$

Performing the change of variables

$$
v=\frac{\beta}{2} \sin 2 t, \quad 0 \leq t<\frac{\pi}{4},
$$

we find

$$
e(v)=\beta m \cos t, \quad e(v)-v e^{\prime}(v)=\beta m \frac{\cos ^{3} t}{\cos 2 t},
$$

while from $e^{\prime}(v)=h(r)$ we get

$$
r=\frac{\cos 2 t}{m \sin t} .
$$

Upon inserting these intermediate results in our principal envelope formula (2), we arrive at the "Martin-Roy-based" Coulomb lower bound

$$
E_{0} \geq \min _{0 \leq t<\pi / 4}\left[\beta m \frac{\cos ^{3} t}{\cos 2 t}+V\left(\frac{\cos 2 t}{m \sin t}\right)\right] .
$$

\section{Concave transform of harmonic-oscillator potential}

For the class of potentials $V(r)$ which are concave transforms $V(r)=g(h(r)), g^{\prime \prime}<0$, of the harmonic-oscillator potential $h(r)=r^{2}$ envelope upper bounds on the eigenvalues $E_{n \ell}$ of the spinless-Salpeter Hamiltonian (1) may be found for all energy levels (cf. Eq. (18) of Ref. [4]):

$$
E_{n \ell} \leq \min _{r>0}\left[\beta \sqrt{m^{2}+\frac{P^{2}}{r^{2}}}+V(r)\right], \quad P \equiv P_{n \ell}(2)=2 n+\ell-\frac{1}{2} .
$$

\section{Concave transform of linear potential}

For the class of potentials $V(r)$ which are concave transforms $V(r)=g(h(r)), g^{\prime \prime}<0$, of the linear potential $h(r)=r$ the "harmonic-oscillator based" envelope upper bounds (17) on the eigenvalues $E_{n \ell}$ of the spinless-Salpeter Hamiltonian (1) may be (significantly) improved by 
considering the composition of the envelope approximations for the kinetic-energy operator and the interaction-potential operator along the following lines. In the present investigation we focus our attention to attractive potentials $V(r)$, that is, to all potentials which exhibit a monotone increase with increasing radial coordinate $r: g^{\prime}>0$. Every function $g(r)$ that is a monotone increasing $\left[g^{\prime}(r)>0\right]$ and concave $\left[g^{\prime \prime}(r)<0\right]$ function of $r$ is a concave function $f\left(r^{2}\right)$ of $r^{2}: g(r)=f\left(r^{2}\right)$ with $f^{\prime \prime}\left(r^{2}\right)<0$. Consequently, all potentials $V(r)$ studied in this section also belong to the class of potentials considered in Sec. 5 .

The square-root operator $k\left(\mathbf{p}^{2}\right) \equiv \sqrt{m^{2}+\mathbf{p}^{2}}$ of the relativistic kinetic energy, regarded as a function of $\mathbf{p}^{2}$, is monotone increasing (i.e., $k^{\prime}\left(\mathbf{p}^{2}\right)>0$ ) and concave (i.e., $\left.k^{\prime \prime}\left(\mathbf{p}^{2}\right)<0\right)$. Let us assume that the interaction potential $V(r)$ exhibits a similar behaviour, that is, that the operator $V(r)=g(r)$ is monotone increasing, i.e., $g^{\prime}(r)>0$, and concave, i.e., $g^{\prime \prime}(r)<0$. These operators are bounded from above by their respective upper-tangent approximations:

$$
\begin{aligned}
& k\left(\mathbf{p}^{2}\right) \leq a_{1}(q)+b_{1}(q) \mathbf{p}^{2}, \\
& g(r) \leq a_{2}(t)+b_{2}(t) r,
\end{aligned}
$$

with

$$
\begin{aligned}
& b_{1}(q)=k^{\prime}(q), \quad a_{1}(q)=k(q)-q k^{\prime}(q), \\
& b_{2}(t)=g^{\prime}(t), \quad a_{2}(t)=g(t)-t g^{\prime}(t),
\end{aligned}
$$

where $\mathbf{p}^{2}=q$ denotes the point of contact between the kinetic-energy operator $k\left(\mathbf{p}^{2}\right)$ and its upper-tangent approximation $a_{1}(q)+b_{1}(q) \mathbf{p}^{2}$, and $r=t$ labels the point of contact between the interaction-potential operator $g(r)$ and its upper-tangent approximation $a_{2}(t)+b_{2}(t) r$. Consequently, our semirelativistic Hamiltonian $H$ in Eq. (1) satisfies the operator inequality

$$
H \equiv \beta k\left(\mathbf{p}^{2}\right)+V(r) \leq \bar{H}(q, t) \equiv \beta\left[a_{1}(q)+b_{1}(q) \mathbf{p}^{2}\right]+a_{2}(t)+b_{2}(t) r .
$$

The eigenvalues $\bar{E}_{n \ell}(q, t)$ of the upper "tangential Hamiltonian" $\bar{H}(q, t)$ are given in terms of the numbers $P_{n \ell}(1)$ listed in Table 1 by the exact formula

$$
\bar{E}_{n \ell}(q, t)=3\left[\frac{\beta b_{1}(q) b_{2}^{2}(t) P_{n \ell}^{2}(1)}{4}\right]^{1 / 3}+\beta a_{1}(q)+a_{2}(t) .
$$

In order to find the best such upper bound, we must minimize with respect to both $q$ and $t$. The critical equations immediately yield the necessary conditions

$$
\frac{1}{2 \beta} \frac{g^{\prime}(\hat{t})}{k^{\prime}(\hat{q})}=\frac{\hat{q}^{3 / 2}}{P_{n \ell}(1)}=\frac{P_{n \ell}^{2}(1)}{\hat{t}^{3}} .
$$

From the second of the above equalities we deduce that, for given quantum numbers $n$ and $\ell$, the minimizing values of the parameters $q$ and $t$ are constrained to the curve $\hat{q} \hat{t}^{2}=P_{n \ell}^{2}(1)$ in the $(q, t)$ parameter plane. On this curve, and with the coefficients (18) of our upper-tangent approximations and the necessary (critical) conditions (20), the minimum of the expression on the right-hand side of the upper energy bound (19) may be cast into a new, simpler form:

$$
\min _{\hat{t}>0}\left[\beta k\left(\frac{P_{n \ell}^{2}(1)}{\hat{t}^{2}}\right)+g(\hat{t})\right] \text {. }
$$

By remembering the above definitions of $k\left(\mathbf{p}^{2}\right)$ and $g(r)$, this expression eventually becomes

$$
E_{n \ell} \leq \min _{r>0}\left[\beta \sqrt{m^{2}+\frac{P^{2}}{r^{2}}}+V(r)\right], \quad P \equiv P_{n \ell}(1) .
$$

These upper bounds on the entire discrete spectrum $\left\{E_{n \ell}\right\}$ are valid for any central potential $V(r)$ which is a monotone increasing and concave function of the linear potential $h(r)=r$. 


\section{Variational upper bounds}

The standard tool for the derivation of rigorous upper bounds on the eigenvalues $E$ of some self-adjoint operator $H$ is the Rayleigh-Ritz variational technique [10,11], which is based on the minimum-maximum principle: If the (discrete) eigenvalues $E_{k}, k=0,1,2, \ldots$, of $H$ are ordered according to $E_{0} \leq E_{1} \leq E_{2} \leq \cdots$, then the first $d$ of them are bounded from above by the $d$ eigenvalues $\widehat{E}_{k}, k=0,1, \ldots, d-1$, ordered according to $\widehat{E}_{0} \leq \widehat{E}_{1} \leq \cdots \leq \widehat{E}_{d-1}$, of that operator $\widehat{H}$ which is obtained by the restriction of $H$ to a $d$-dimensional subspace $D_{d}$ of the domain of $H$, that is, $E_{k} \leq \widehat{E}_{k}$ for all $k=0,1, \ldots, d-1$. Hence, for the accuracy of the obtained results, an appropriate, "reasonable" definition of this trial subspace $D_{d}$ is crucial. If this $d$-dimensional subspace $D_{d}$ is spanned by a chosen set of $d$ linearly independent basis vectors $\left|\psi_{k}\right\rangle, k=0,1, \ldots, d-1$, the set of eigenvalues $\widehat{E}$ may immediately be determined by diagonalizing the $d \times d$ matrix $\left(\left\langle\psi_{i}|\widehat{H}| \psi_{j}\right\rangle\right), i, j=0,1, \ldots, d-1$, that is, as the $d$ roots of the characteristic equation

$$
\operatorname{det}\left(\left\langle\psi_{i}|\widehat{H}| \psi_{j}\right\rangle-\widehat{E}\left\langle\psi_{i} \mid \psi_{j}\right\rangle\right)=0, \quad i, j=0,1, \ldots, d-1 .
$$

The relativistic virial theorem derived in Ref. [18] — for a very comprehensive review, see Ref. [19] - allows to define a precise quantitative measure [8, 9] for the quality of the results found within the framework of variational techniques: according to the analysis presented in Refs. [8, 9], for some generic Hamiltonian operator $H$ consisting of a momentum-dependent kinetic-energy term $T(\mathbf{p})$ and a coordinate-dependent interaction-potential term $V(\mathbf{x})$, i.e.,

$$
H=T(\mathbf{p})+V(\mathbf{x}),
$$

the accuracy of trial states $|\varphi\rangle$ which approximate the exact bound state under study can be quantitatively estimated by the deviation from zero of the quantity (called $\nu$ in Refs. [8, 9])

$$
Q \equiv 1-\frac{\left\langle\varphi\left|\mathbf{p} \cdot \frac{\partial}{\partial \mathbf{p}} T(\mathbf{p})\right| \varphi\right\rangle}{\left\langle\varphi\left|\mathbf{x} \cdot \frac{\partial}{\partial \mathbf{x}} V(\mathbf{x})\right| \varphi\right\rangle}(\equiv-\nu) .
$$

The main advantage of this measure for the accuracy of approximate eigenstates $|\varphi\rangle$ is that it does not require any information on the solutions of the investigated eigenvalue problem other than the one provided by the variational approximation technique itself.

In order to get a first idea of the position of the lowest energy level $E_{0}$, a one-dimensional trial space is sufficient; a basis for the latter might be any of the two-parameter (normalized) trial functions

$$
\phi(\mathbf{x})=\sqrt{\frac{n \alpha^{3 / n}}{4 \pi \Gamma\left(\frac{3}{n}\right)}} \exp \left(-\frac{\alpha}{2} r^{n}\right) .
$$

Here the scale parameter $\alpha>0$ is varied. The parameter $n>0$ allows for later optimization. For the sake of definiteness, let us consider an interaction potential $V(r)$ which is the sum of (attractive) pure power-law terms $a(q) \operatorname{sgn}(q) r^{q}$ with $q \neq 0$ and a logarithmic term $a(0) \ln r$ :

$$
V(r)=\sum_{q \neq 0} a(q) \operatorname{sgn}(q) r^{q}+a(0) \ln r .
$$

We assume, of course, that the potential coefficients $a(q) \geq 0$ are not all zero. With the help of Jensen's inequality [20], and by a tricky redefinition of the scale variable $\alpha$, the variational upper bound on the ground-state eigenvalue $E_{0}$ of the Hamiltonian (1), resulting from this choice of $\phi(\mathbf{x})$ as basis vector of our one-dimensional trial space, may be cast into the form

$$
E_{0} \leq \min _{r>0}\left[\beta \sqrt{m^{2}+\frac{1}{r^{2}}}+\sum_{q \neq 0} a(q) \operatorname{sgn}(q)(\mathcal{P}(n, q) r)^{q}+a(0) \ln (\mathcal{P}(n, 0) r)\right],
$$


with the abbreviations

$$
\begin{aligned}
& \mathcal{P}(n, q)=\frac{n}{2}\left(\frac{\Gamma\left(2+\frac{1}{n}\right)}{\Gamma\left(\frac{3}{n}\right)}\right)^{1 / 2}\left(\frac{\Gamma\left(\frac{q+3}{n}\right)}{\Gamma\left(\frac{3}{n}\right)}\right)^{1 / q}, \quad q \neq 0, \\
& \mathcal{P}(n, 0)=\frac{n}{2}\left(\frac{\Gamma\left(2+\frac{1}{n}\right)}{\Gamma\left(\frac{3}{n}\right)}\right)^{1 / 2} \exp \left(\frac{1}{n} \psi\left(\frac{3}{n}\right)\right),
\end{aligned}
$$

where $\psi$ denotes the digamma function

$$
\psi(z) \equiv \frac{1}{\Gamma(z)} \frac{\mathrm{d} \Gamma(z)}{\mathrm{d} z} .
$$

\section{The "Laguerre" trial space}

Unless the eigenstate corresponding to some eigenvalue $E_{k}$ is already an element of the trial space $D_{d}$, any upper bounds on the eigenvalues of an operator bounded from below may be improved by enlarging $D_{d}$ to higher $d$, or by spanning $D_{d}$ by more sophisticated basis states.

For the class of spherically symmetric potentials $V(r)$ studied here, a very popular choice for the basis states which span the $d$-dimensional trial space $D_{d}$ of the variational technique are "Laguerre" trial states, given in their configuration-space representation by $[21,22,6,7]$

$$
\psi_{k, \ell m}(\mathbf{x})=\sqrt{\frac{(2 \mu)^{2 \ell+2 \rho+1} k !}{\Gamma(2 \ell+2 \rho+k+1)}} r^{\ell+\rho-1} \exp (-\mu r) L_{k}^{(2 \ell+2 \rho)}(2 \mu r) \mathcal{Y}_{\ell m}\left(\Omega_{\mathbf{x}}\right) .
$$

Here $L_{k}^{(\gamma)}(x)$ are the generalized Laguerre polynomials (for the parameter $\gamma$ ) [15], defined by the power series

$$
L_{k}^{(\gamma)}(x)=\sum_{t=0}^{k}(-1)^{t}\left(\begin{array}{c}
k+\gamma \\
k-t
\end{array}\right) \frac{x^{t}}{t !}
$$

and orthonormalized, with the weight function $x^{\gamma} \exp (-x)$, according to

$$
\int_{0}^{\infty} \mathrm{d} x x^{\gamma} \exp (-x) L_{k}^{(\gamma)}(x) L_{k^{\prime}}^{(\gamma)}(x)=\frac{\Gamma(\gamma+k+1)}{k !} \delta_{k k^{\prime}} ;
$$

$\mathcal{Y}_{\ell m}(\Omega)$ are the spherical harmonics for angular momentum $\ell$ and projection $m$, depending on the solid angle $\Omega$ and orthonormalized according to

$$
\int \mathrm{d} \Omega \mathcal{Y}_{\ell m}^{*}(\Omega) \mathcal{Y}_{\ell^{\prime} m^{\prime}}(\Omega)=\delta_{\ell \ell^{\prime}} \delta_{m m^{\prime}}
$$

The trial functions (23) involve two variational parameters, $\mu$ (with the dimension of mass) and $\rho$ (dimensionless), which, by the requirement of normalizability of these functions, are subject to the constraints $\mu>0$ and $2 \rho>-1$.

One of the main advantages of the choice (23) for the variational trial states is the easy availability of an analytic expression for their momentum-space representation, obtained by Fourier transformation of $\psi_{k, \ell m}(\mathbf{x})$ :

$$
\begin{aligned}
\widetilde{\psi}_{k, \ell m}(\mathbf{p}) & =\sqrt{\frac{(2 \mu)^{2 \ell+2 \rho+1} k !}{\Gamma(2 \ell+2 \rho+k+1)}} \frac{(-\mathrm{i})^{\ell}|\mathbf{p}|^{\ell}}{2^{\ell+1 / 2} \Gamma\left(\ell+\frac{3}{2}\right)} \sum_{t=0}^{k} \frac{(-1)^{t}}{t !}\left(\begin{array}{c}
k+2 \ell+2 \rho \\
k-t
\end{array}\right) \\
& \times \frac{\Gamma(2 \ell+\rho+t+2)(2 \mu)^{t}}{\left(\mathbf{p}^{2}+\mu^{2}\right)^{(2 \ell+\rho+t+2) / 2}} F\left(\frac{2 \ell+\rho+t+2}{2},-\frac{\rho+t}{2} ; \ell+\frac{3}{2} ; \frac{\mathbf{p}^{2}}{\mathbf{p}^{2}+\mu^{2}}\right) \\
& \times \mathcal{Y}_{\ell m}\left(\Omega_{\mathbf{p}}\right),
\end{aligned}
$$


with the hypergeometric series $F$, defined by

$$
F(u, v ; w ; z)=\frac{\Gamma(w)}{\Gamma(u) \Gamma(v)} \sum_{n=0}^{\infty} \frac{\Gamma(u+n) \Gamma(v+n)}{\Gamma(w+n)} \frac{z^{n}}{n !} .
$$

The choice of the Laguerre states as the basis of the trial space $D_{d}$ allows to calculate the matrix elements of (any linear combination of) pure power-law potentials $V(r)=a(q) r^{q}$ and under certain circumstances (for instance, for $\mu=m$ for either radial or orbital excitations) the matrix elements of the kinetic term on purely analytical grounds, and to arrive therefore at analytical expressions for the $d \times d$ Hamiltonian matrix $\left(\left\langle\psi_{i}|\widehat{H}| \psi_{j}\right\rangle\right), i, j=0,1, \ldots, d-1$. The explicit algebraic expressions of these matrix elements may be found in Refs. [22, 6, 7]. Up to and including a trial-space dimension $d=4$, the Hamiltonian matrix $\left(\left\langle\psi_{i}|\widehat{H}| \psi_{j}\right\rangle\right)$ may be diagonalized algebraically. For the funnel potential (14), the case $d=1$ yields the bound

$$
E_{0} \leq\left(\frac{64 \beta}{15 \pi}-c_{1}\right) m+\frac{3 c_{2}}{2 m} .
$$

\section{The "local-energy" theorem}

Some information on both upper and lower bounds on the isolated eigenvalues $E_{n \ell}$ of some Hamiltonian $H$ may be gained with the help of the so-called "local-energy" theorem $[23,11]$. Unfortunately, the proof of this fundamental criterion makes use of the "nodal theorem" for the eigenstates of $H$. As a consequence of this, for Hamiltonians $H$ defined in more than one dimension the local-energy theorem can be applied only to the ground state of the spectrum. It has been applied to the (three-dimensional) "relativistic Coulomb problem" in Ref. [24].

Upper bounds on the energy levels of Hamiltonians bounded from below can be obtained with considerably more efficiency by the variational technique described in Sec. 7 . Therefore we employ here the local-energy theorem for the derivation of lower bounds on the spectrum of $H$. In order to formulate the local-energy theorem in momentum-space representation, we introduce the "local energy"

$$
\mathcal{E}(\mathbf{p}) \equiv \beta \sqrt{m^{2}+\mathbf{p}^{2}}+\frac{\int \mathrm{d}^{3} q \tilde{V}(\mathbf{p}-\mathbf{q}) \phi(\mathbf{q})}{\phi(\mathbf{p})} .
$$

Here, $\widetilde{V}(\mathbf{p})$ is the Fourier transform of the interaction potential $V(\mathbf{x})$ under consideration:

$$
\widetilde{V}(\mathbf{p}) \equiv \frac{1}{(2 \pi)^{3}} \int \mathrm{d}^{3} x \exp (-\mathrm{ip} \cdot \mathbf{x}) V(\mathbf{x}) .
$$

$\phi(\mathbf{p})$ denotes a suitably chosen, positive trial function: $\phi(\mathbf{p})>0$. The "lower-bound part" of the local-energy theorem then states that the lowest-lying eigenvalue $E_{0}$ of the Hamiltonian $H$ is bounded from below by the infimum of $\mathcal{E}(\mathbf{p})$ with respect to the momentum variable $\mathbf{p}$ :

$$
E_{0} \geq \inf _{\mathbf{p}} \mathcal{E}(\mathbf{p})
$$

The application of the local-energy theorem to confining potentials demands special care for the following reason: the Fourier transform of any confining potential is not well-defined; the potential has to be regarded as a distribution and an appropriate regularization must be applied. For instance, the linear potential $V_{\mathrm{L}}(r)=\lambda r$, where $\lambda$ is a coupling parameter with mass dimension 2, may be regularized by the $\operatorname{exponential~} \exp (-\varepsilon r), \varepsilon \geq 0$, or by writing it as the derivative of this exponential or as the second derivative of a Yukawa-type function:

$$
V_{\mathrm{L}}(r)=\lambda r=\lambda \lim _{\varepsilon \downarrow 0} r \exp (-\varepsilon r)=-\lambda \lim _{\varepsilon \downarrow 0} \frac{\partial}{\partial \varepsilon} \exp (-\varepsilon r)=\lambda \lim _{\varepsilon \downarrow 0} \frac{\partial^{2}}{\partial \varepsilon^{2}} \frac{\exp (-\varepsilon r)}{r} .
$$


With these regularizations, the Fourier transform $\tilde{V}_{\mathrm{L}}(\mathbf{p})$ of the linear potential $V_{\mathrm{L}}(r)$ reads

$$
\widetilde{V}_{\mathrm{L}}(\mathbf{p})=\widetilde{V}_{\mathrm{L}}(|\mathbf{p}|)=-\frac{\lambda}{\pi^{2}} \lim _{\varepsilon \downarrow 0} \frac{\mathbf{p}^{2}-3 \varepsilon^{2}}{\left(\mathbf{p}^{2}+\varepsilon^{2}\right)^{3}} .
$$

Note that (as a consistency check) the integral of the Fourier transform $\widetilde{V}_{\mathrm{L}}(\mathbf{p})$ has to vanish: $\int \mathrm{d}^{3} p \widetilde{V}_{\mathrm{L}}(\mathbf{p})=0$. This holds for every power-law potential $V(r)=a_{n} r^{n}$ with exponent $n>0$. The singularity structure of $\widetilde{V}_{\mathrm{L}}(\mathbf{p})$ becomes manifest when writing the derivative in $V_{\mathrm{L}}(r)$ as a difference quotient,

$$
V_{\mathrm{L}}(r)=\lambda r=-\lambda \lim _{\varepsilon \downarrow 0} \frac{\exp (-\varepsilon r)-1}{\varepsilon}
$$

which yields

$$
\widetilde{V}_{\mathrm{L}}(\mathbf{p})=\widetilde{V}_{\mathrm{L}}(|\mathbf{p}|)=\lambda \lim _{\varepsilon \downarrow 0}\left[\frac{1}{\varepsilon} \delta^{(3)}(\mathbf{p})-\frac{1}{\pi^{2}\left(\mathbf{p}^{2}+\varepsilon^{2}\right)^{2}}\right] .
$$

Thus the Fourier transform $\tilde{V}_{\mathrm{L}}(\mathbf{p})$ of the linear potential consists of a (negative) regular part and a singular part, which is a distribution localized at the origin $\mathbf{p}=\mathbf{0}$. However, a detailed inspection shows that for the case of the funnel potential the momentum-space ground-state eigenfunction is indeed positive. This fact justifies our use of a positive trial function $\phi(\mathbf{p})$.

The simplest normalizable trial function which comes to one's mind is the exponential:

$$
\phi(r)=\sqrt{\frac{\mu^{3}}{\pi}} \exp (-\mu r)
$$

This choice for the ground-state trial function $\phi(r)$ corresponds to the case $k=\ell=m=0$ and $\rho=1$ of the Laguerre basis functions $\psi_{k, \ell m}(\mathbf{x})$ introduced in Sec. 8. It is straightforward to calculate the Fourier transform of $\phi(r)$, or to extract it from the general expression given explicitly in Sec. 8:

$$
\phi(\mathbf{p})=\phi(|\mathbf{p}|)=\frac{\sqrt{2 \mu^{3}}}{\pi|\mathbf{p}|\left(\mathbf{p}^{2}+\mu^{2}\right)} \sin \left(2 \arctan \frac{|\mathbf{p}|}{\mu}\right)=\frac{2 \sqrt{2 \mu^{5}}}{\pi\left(\mathbf{p}^{2}+\mu^{2}\right)^{2}} .
$$

In order to illustrate the application of the local-energy theorem, let us consider the example of a potential $V(r)$ which is a sum of pure power-law terms:

$$
V(r)=\sum_{q} a(q) r^{q}
$$

For this power-law potential and the ground-state trial function $\phi(r)$, it is straightforward to obtain the corresponding local energy $\mathcal{E}(\mathbf{p})$ by Fourier transformation of the product $r^{q} \phi(r)$ :

$$
\mathcal{E}(\mathbf{p})=\beta \sqrt{m^{2}+\mathbf{p}^{2}}+\sum_{q} \frac{a(q) \Gamma(q+2)}{2 \mu|\mathbf{p}|\left(\mathbf{p}^{2}+\mu^{2}\right)^{q / 2-1}} \sin \left[(q+2) \arctan \frac{|\mathbf{p}|}{\mu}\right] .
$$

The variational parameter $\mu$ entering into our ground-state trial function $\phi(r)$ either may be given a chosen fixed value, or it may be adjusted in order to maximize the lower bound (24).

\section{Sums of distinct potential terms}

For the class of potentials $V(r)$ which consist of two or more distinct terms $V^{(i)}(r) \neq V^{(j)}(r)$ for $i \neq j$, where, for every single component problem defined by the "one-term" Hamiltonian $H_{i} \equiv \beta \sqrt{m^{2}+\mathbf{p}^{2}}+V^{(i)}(r)$, information about the bottom of its spectrum is available, these individual pieces of information may be combined to form a lower bound on the spectrum of the Hamiltonian (1) with such a sum potential $V(r)$. The precise requirements for this "sum approximation" to work are the following: 
1. The interaction potential $V(r)$ entering in the semirelativistic Hamiltonian of Eq. (1) is a sum of (more than one) different components $V^{(i)}(r)=c_{i} h^{(i)}(r)$ :

$$
V(r)=\sum_{i} c_{i} h^{(i)}(r)
$$

2. Every component problem $H_{i} \equiv \beta \sqrt{m^{2}+\mathbf{p}^{2}}+c_{i} h^{(i)}(r)$ supports, for sufficiently large values of its coupling parameter $c_{i}$, a discrete eigenvalue at the bottom of its spectrum.

The sum approximation has already been studied for the case of Schrödinger Hamiltonians (involving nonrelativistic kinetic energies) in Refs. $[1,25]$. The analysis of the corresponding semirelativistic problem has been presented in full generality in Ref. [26]. Therefore, here we summarize only the result for the case in which the potential $V(r)$ is a sum of attractive pure power-law terms:

$$
V(r)=\sum_{q \neq 0} a(q) \operatorname{sgn}(q) r^{q}
$$

where the coupling parameters $a(q)$ are non-negative and not all zero. For this potential, a lower bound to the bottom of the spectrum of the semirelativistic Hamiltonian (1), which is, by assumption, the lowest eigenvalue $E_{0}$ of $H$, is given by the expression

$$
E_{0} \geq \min _{r>0}\left[\beta \sqrt{m^{2}+\frac{1}{r^{2}}}+\sum_{q \neq 0} a(q) \operatorname{sgn}(q)(P(q) r)^{q}\right],
$$

if, for the particular potential $V(r)$ under consideration, there exist (or there can be found) $P$ numbers $P(q)$ such that, whenever there is only one term present in the potential (25), the right-hand side of the inequality (26) yields either the exact ground-state energy eigenvalue of the corresponding "single-term" problem or a lower bound to it.

Consequently, when applying our general sum lower bound (26) to a given sum potential $V(r)$, the main task is to derive appropriate $P$ numbers for all components in the sum (25). Let us illustrate this by considering the two examples relevant for the funnel potential (14).

- For a Coulomb component potential $h^{(i)}(r)=-1 / r$, corresponding to the case $q=-1$ in Eq. (25), we may take advantage from the explicit results presented in Sec. 4. By the change of variables $r \rightarrow \operatorname{Pr}$, the Coulomb lower bound (12) may be cast into the form

$$
E_{0} \geq \min _{r>0}\left[\beta \sqrt{m^{2}+\frac{1}{r^{2}}}+V(P r)\right], \quad v<\beta v_{P} .
$$

Specifying this general result to the Coulomb potential $V(r)=-v / r$ yields the bound

$$
E_{0} \geq \min _{r>0}\left[\beta \sqrt{m^{2}+\frac{1}{r^{2}}}-\frac{v}{P r}\right], \quad v<\beta v_{P} .
$$

This bound is, of course, nothing else but, for $P=2 / \pi$, the Herbst lower bound (9) or, for arbitrary $P$, the Herbst-like lower bound (11) to the relativistic Coulomb problem. Consequently, for the Coulomb component term we may identify the $P$ number $P(-1)$ required for the lower bound (26) with the lower-bound parameter $P$ defined in Sec. 4 : $P(-1)=P$.

- For a linear component potential $h^{(i)}(r)=r$, that is, for the case $q=1$ in Eq. (25), (a possible value of) the $P$ number $P(1)$ required for the lower bound (26) turns out to be related to the ground-state eigenvalue $e_{0}(v)$ of the Hamiltonian operator $\sqrt{\mathbf{p}^{2}}+v r$ by

$$
e_{0}(v)=2 \sqrt{v P(1)}
$$

a numerical determination of this lowest energy eigenvalue $e_{0}(v)$ yields $P(1)=1.2457$. 


\section{Comparison: The case of the funnel potential}

In order to demonstrate the power of the different energy bounds derived so far, let us apply the above results to the very illustrative example of the Coulomb-plus-linear potential (14). By factorizing off an overall coupling constant $0<v \leq 1$, we write this potential in the form

$$
V(r)=v\left(-\frac{a}{r}+b r\right) .
$$

For the coupling parameters entering in this funnel potential $V(r)$, different notations have been used in Eqs. (14), (25), and (27). Evidently, the three sets of potential coefficients have to be identified according to $a(-1) \equiv c_{1} \equiv a v>0$ and $a(1) \equiv c_{2} \equiv b v>0$.

Fig. 1 compares the various energy bounds derived or investigated in Secs. 4 through 10 for the ground-state energy eigenvalue $E_{0}$ of the Hamiltonian (1) with the funnel potential $(27)$ as a function of the overall coupling parameter $v$. These energy bounds necessarily form a bunch of concave curves $E(v)$, all starting at the (free-energy) value $E_{0}(v=0)=\beta m=1$.

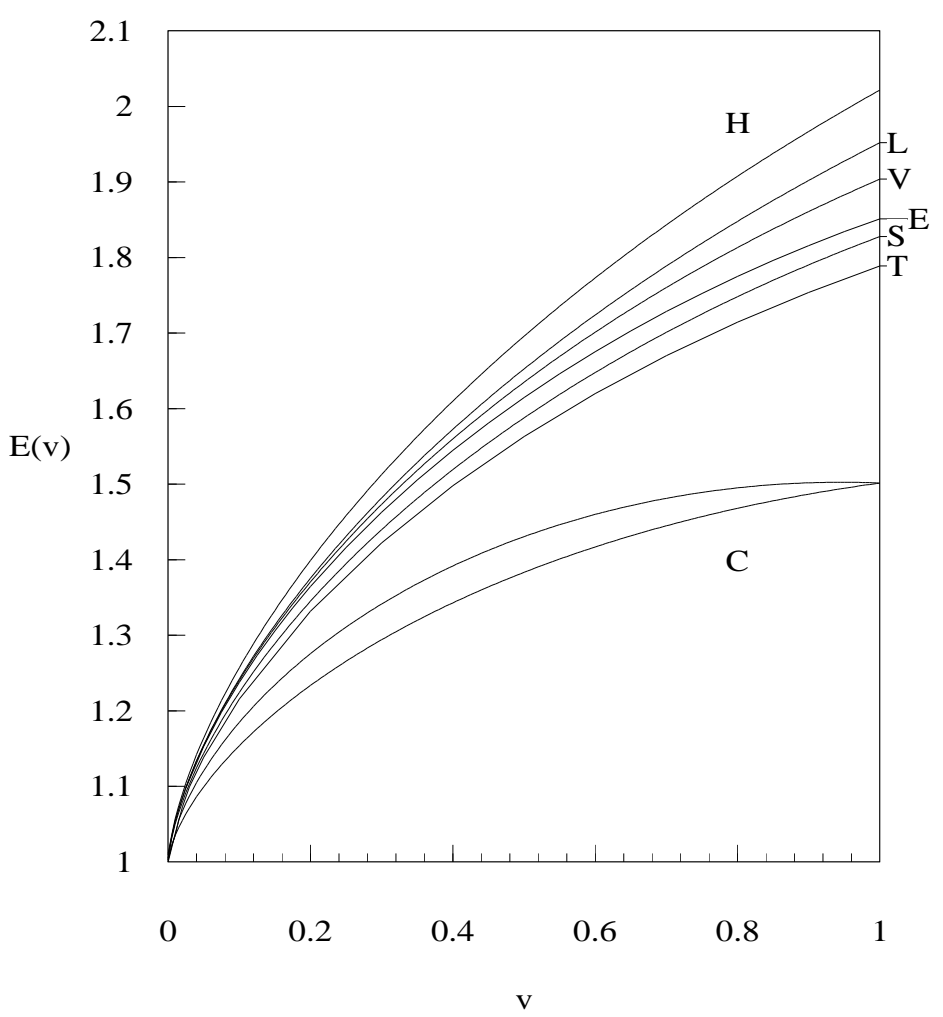

Figure 1: Comparison of bounds to the ground-state $[(n, \ell)=(1,0)]$ energy eigenvalue of the semirelativistic Hamiltonian $H=\beta \sqrt{m^{2}+\mathbf{p}^{2}}+V(r)$ with a Coulomb-plus-linear potential $V(r)=v(-a / r+b r)$, for $a=0.2, b=0.5, m=\beta=1$. The bounds are plotted as functions $E(v)$ of the overall coupling parameter $v$ in $V(r)$. The energy bounds compared here include (from top to bottom) the harmonic-oscillator upper bound (H) of Eq. (17), the linear upper bound (L) of Eq. (21), the "one-dimensional" variational upper bound (V) of Eq. (22) with $n=1.74$, the variational upper bound (E) based on a 25-dimensional Laguerre trial space, which should come rather close to the exact eigenvalue, the sum lower bound (S) of Eq. (26) with $P$ numbers $P(-1)=0.72811$ and $P(1)=1.2457$, the lower bound $(\mathrm{T})$ provided by the local-energy theorem (24), and two Coulomb lower bounds (C) according to Eq. (12), where with respect to the parameter $P$ the lower curve corresponds to the fixed value $P=0.72811$ whereas the upper curve corresponds to the optimized value of $P$ calculated from Eq. (16). 
The Coulomb lower bound (12) may be applied to the funnel potential either with a fixed value of the lower-bound parameter $P$ or with the optimized $P(v)$ computed from Eq. (16). For "fixed- $P$ " bounds, the maximum value of $P$ allowed by the Coulomb coupling constant constraint discussed in Sec. 4 is found, for given values of the kinetic-term parameters $\beta, m$ and potential coefficients $c_{1}, c_{2}$, as solution of that relation which is obtained when equating the left-hand and right-hand sides of the inequality (15) with the critical coupling $v_{P}$ given by the definition (13), that is, by solving, for the maximum values of $c_{1}$ and $c_{2}$ occuring here, the constraint (16):

$$
c_{1}+\frac{P^{4}}{1-P^{2}} \frac{c_{2}}{m^{2}}=\beta P \sqrt{1-P^{2}} .
$$

For a single, unit-mass particle, i.e., $\beta=1$ and $m=1$, and the values of the funnel-potential coupling parameters $a$ and $b$ used in Fig. $1, a=0.2$ and $b=0.5$, this yields $P=0.728112$.

We try to approach the state we are interested in as closely as possible by the application of the Rayleigh-Ritz variational technique briefly sketched in Sec. 7, with the trial space $D_{d}$ spanned by the Laguerre basis defined in Sec. 8. By fixing the variational parameter $\mu$ to the value $\mu=m$, we are able to obtain the matrix elements of the Hamiltonian (1) analytically, avoiding thereby the necessity to use a numerical integration procedure for the evaluation of these matrix elements; for definiteness, we fix the variational parameter $\rho$ to the value $\rho=1$.

The accuracy of such an approximation to an eigenstate may be estimated with the help of the "virial-theorem-inspired" quality measure $Q$ discussed in Sec. 7. Fig. 2 shows, for the trial-space dimension $d=25$ chosen for the variational bound (E) in Fig. 1, the dependence of the accuracy $Q$ on the overall coupling $v$. We obtain $Q=|Q| \leq 1.1 \times 10^{-4}$ over the whole parameter range $0<v \leq 1$. Such high accuracy should be sufficient for the present purpose.

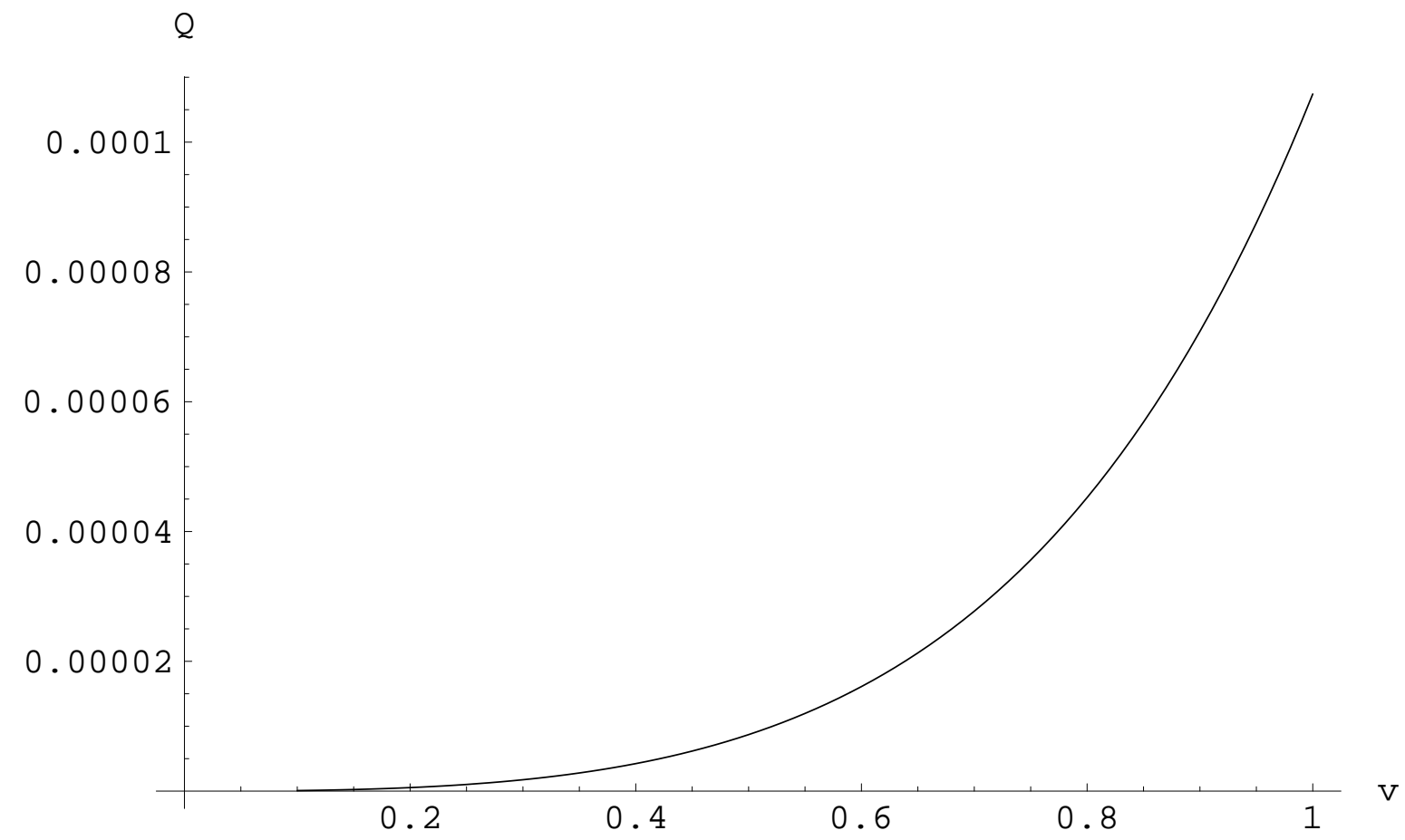

Figure 2: Measure $Q$ of the accuracy of the (variational) approximation of the ground-state eigenfunction of the semirelativistic Hamiltonian of Fig. 1 [that is, $H=\beta \sqrt{m^{2}+\mathbf{p}^{2}}+V(r)$, where $V(r)$ is the Coulomb-plus-linear potential $V(r)=v(-a / r+b r)$ with $a=0.2, b=0.5$, $m=\beta=1$ ] by a superposition of 25 Laguerre basis functions (23) with $\mu=m$ and $\rho=1$. 
For the sake of completeness, we plot, in Fig. 3, the behaviour of the ground state of the Hamiltonian (1) with the Coulomb-plus-linear potential (27), for the maximum value $v=1$ of the overall coupling parameter $v$, determined by the variational technique of Sec. 7 , with the Laguerre basis of Sec. 8, in both configuration and momentum space. As expected, the ground-state momentum-space eigenfunction possesses no node, which is in agreement with our assumption of a positive momentum-space trial function $\phi(\mathbf{p})$ (which is also depicted, as the case $d=1$, in Fig. 3) in the derivation of the local-energy lower energy bound in Sec. 9.

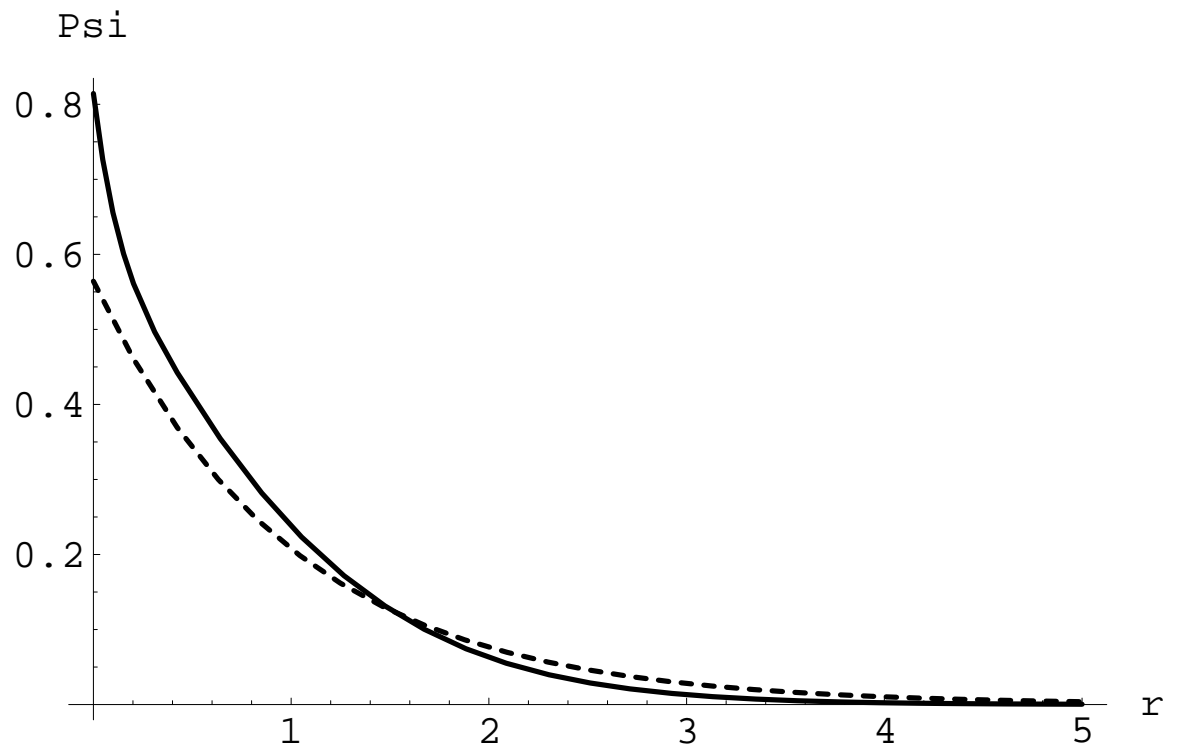

(a)

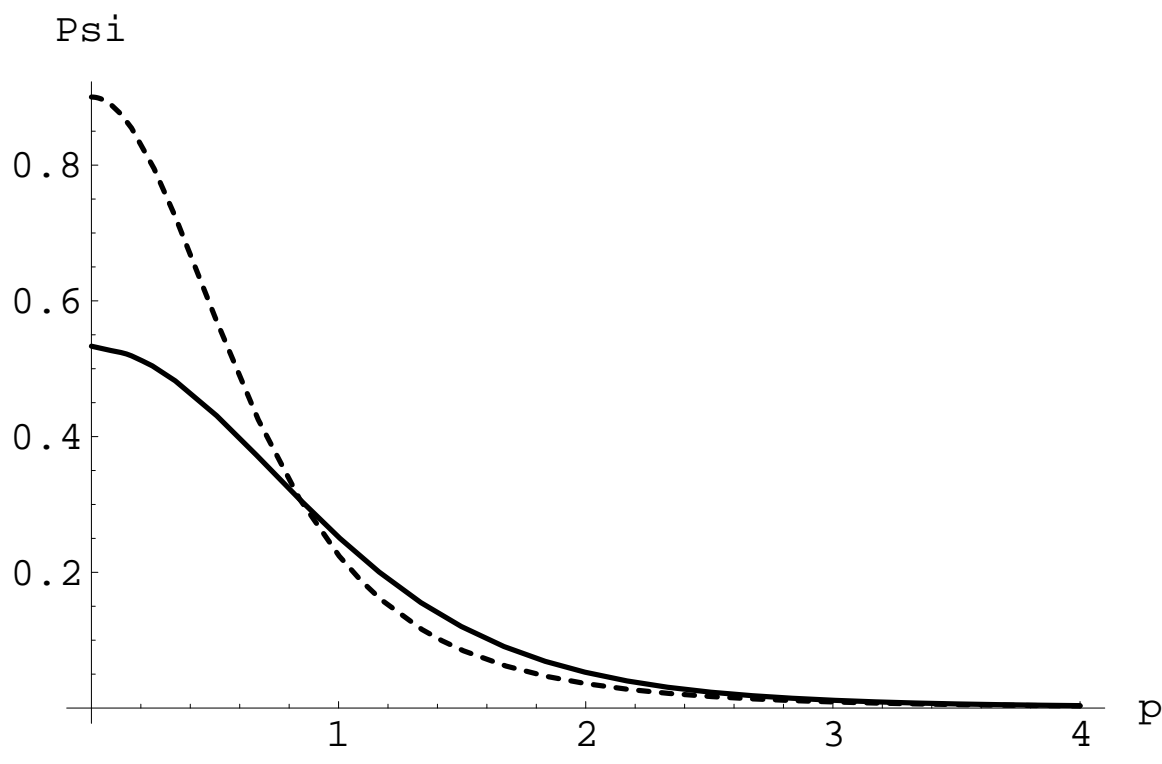

(b)

Figure 3: Variational approximation of the lowest-lying eigenstate in configuration space (a) and momentum space (b) of the semirelativistic Hamiltonian (1), $H=\beta \sqrt{m^{2}+\mathbf{p}^{2}}+V(r)$, with a Coulomb-plus-linear potential $V(r)=-a / r+b r$, for $a=0.2, b=0.5$ and $m=\beta=1$, by a superposition of Laguerre basis functions (23) with the variational parameters fixed to $\mu=m$ and $\rho=1$, for the trial-space dimensions $d=25$ (full lines) and $d=1$ (dashed lines). 


\section{Summary and conclusions}

This review of the discrete spectra of semirelativistic Hamiltonians $H$ of the form (1) should be considered as a compilation of rigorous (semi-)analytical results for both upper and lower bounds on the discrete eigenvalues $E_{n \ell}$ of $H$, presented in a way suitable for immediate use: the algebraic structure and the convexity properties with respect to suitable basis potentials $h(r)$ of the interaction potential $V(r)$ one wishes to use in one's semirelativistic Hamiltonian (1) unambiguously determine which of all the energy bounds discussed here actually apply. With the explicit analytical expressions for these bounds on the discrete eigenvalues at one's disposal, one is left with the (numerical) optimization with respect to a single real variable. Clearly, within the respective limitations, the Rayleigh-Ritz variational technique of Secs. 7 and 8 as well as the local-energy theorem of Sec. 9 apply to arbitrary reasonable potentials.

For the particular example considered in Sec. 11, viz., the Coulomb-plus-linear potential (27), the sum lower bound turns out to be somewhat better than the lower bound provided by the local-energy theorem. Clearly, the sum lower bound cannot always be better than the local-energy bound because one could, in principle, choose in the local-energy theorem (24) the exact eigenfunction for the trial function $\phi$ and thus recover the exact energy eigenvalue.

In conclusion, let us stress that all but two of the various energy bounds we have studied in this work may eventually be expressed by essentially the same general semi-classical form. We can view this in the following way: Since the square of the momentum $\mathbf{p}^{2}$ scales like $1 / r^{2}$, we expect that

$$
\left\langle\mathbf{p}^{2}\right\rangle=\frac{P^{2}}{r^{2}}
$$

where $P$ is a suitable coefficient; thus the balance between the kinetic and potential energies required by the minimum-maximum principle might lead to a search for the minimum of an energy expression of the form

$$
\sqrt{m^{2}+\frac{P^{2}}{r^{2}}}+V(r)
$$

What makes this heuristic argument interesting is the fact that definite values of $P$ can be prescribed so that this minimum provides a priori a bound on the (unknown) exact energy. In the case where $V(r)$ is a sum of terms, a trivial change of variables is used to move the $P$ parameters inside the potential $V(r)$, and then optimal distinct parameters can be used for each potential term.

The lower bound by the sum approximation incorporates our best known lower bounds for the sub-problems containing only a single potential term; the final result is the optimized mixture of these contributions. The lower bound by the local-energy theorem (24) could, in principle, achieve arbitrarily high accuracy. The difficulty here, as with variational methods, is to find a suitable trial function. To the extent that we have searched we did not manage to find a better lower bound with the help of the local-energy theorem (24) than that provided immediately by the sum approximation (26).

Our most accurate results overall were upper bounds obtained by using a 25-dimensional trial space spanned by Laguerre functions. We estimate the error for these results to be less than $0.01 \%$ over the total range of potential parameters studied; such accurate estimates are invaluable for estimating the effectiveness of the large variety of alternative simpler energy bounds which we have reviewed in this article. 


\section{References}

[1] R. L. Hall, J. Math. Phys. 24, 324 (1983).

[2] R. L. Hall, J. Math. Phys. 25, 2708 (1984).

[3] R. L. Hall, W. Lucha, and F. F. Schöberl, J. Phys. A 34, 5059 (2001), hep-th/0012127.

[4] R. L. Hall, W. Lucha, and F. F. Schöberl, Int. J. Mod. Phys. A 17, 1931 (2002), hep-th/0110220.

[5] W. Lucha and F. F. Schöberl, Phys. Rev. A 54, 3790 (1996), hep-ph/9603429.

[6] W. Lucha and F. F. Schöberl, Int. J. Mod. Phys. A 14, 2309 (1999), hep-ph/9812368.

[7] W. Lucha and F. F. Schöberl, Fizika B 8, 193 (1999), hep-ph/9812526.

[8] W. Lucha and F. F. Schöberl, Phys. Rev. A 60, 5091 (1999), hep-ph/9904391.

[9] W. Lucha and F. F. Schöberl, Int. J. Mod. Phys. A 15, 3221 (2000), hep-ph/9909451.

[10] M. Reed and B. Simon, Methods of Modern Mathematical Physics IV: Analysis of Operators (Academic Press, New York, 1978).

[11] W. Thirring, A Course in Mathematical Physics 3: Quantum Mechanics of Atoms and Molecules (Springer, New York/Wien, 1990).

[12] W. Lucha and F. F. Schöberl, J. Math. Phys. 41, 1778 (2000), hep-ph/9905556.

[13] A. Martin, Phys. Lett. B 214, 561 (1988).

[14] I. M. Gelfand and S. V. Fomin, Calculus of Variations (Prentice-Hall, Englewood Cliffs, 1963).

[15] Handbook of Mathematical Functions, edited by M. Abramowitz and I. A. Stegun (Dover, New York, 1964).

[16] I. W. Herbst, Commun. Math. Phys. 53, 285 (1977); 55, 316 (1977) (addendum).

[17] A. Martin and S. M. Roy, Phys. Lett. B 233, 407 (1989).

[18] W. Lucha and F. F. Schöberl, Phys. Rev. Lett. 64, 2733 (1990).

[19] W. Lucha and F. F. Schöberl, Mod. Phys. Lett. A 5, 2473 (1990).

[20] W. Feller, An introduction to probability theory and its applications, Volume II (John Wiley, New York, 1971).

[21] S. Jacobs, M. G. Olsson, and C. Suchyta III, Phys. Rev. D 33, 3338 (1986); 34, 3536 (1986) (E).

[22] W. Lucha and F. F. Schöberl, Phys. Rev. A 56, 139 (1997), hep-ph/9609322.

[23] R. J. Duffin, Phys. Rev. 71, 827 (1947);

M. F. Barnsley, J. Phys. A 11, 55 (1978);

B. Baumgartner, J. Phys. A 12, 459 (1979). 
[24] J. C. Raynal, S. M. Roy, V. Singh, A. Martin, and J. Stubbe, Phys. Lett. B 320, 105 (1994).

[25] R. L. Hall, Phys. Rev. D 37, 540 (1988); J. Math. Phys. 33, 1710 (1992).

[26] R. L. Hall, W. Lucha, and F. F. Schöberl, math-ph/0208042, J. Math. Phys. (in print). 